\title{
THE COMPARISON OF MEASURED AND SIMULATED DYNAMIC RESPONSES OF VEHICLES INDICATED BY ROAD PAVEMENT UNEVENNESS
}

\author{
Martin DECKÝ ${ }^{1, *}$, Matúš KOVÁČ ${ }^{1}$, Peter KOTEK ${ }^{1}$ \\ ${ }^{1}$ Department of Highway Engineering, Faculty of Civil Engineering, University of Žilina, Univerzitná \\ 8215/1, 01026 Žilina. \\ corresponding author: martin.decky@fstav.uniza.sk.
}

\section{Abstract}

The article presents the comparison of measured and simulated dynamic responses of heavy vehicle which are indicated by road pavement unevenness. This unevenness was represented by speed control bumps, potholes and stochastic pavement unevenness. The objective simulations were realized through the medium QuarterCar Simulation by means of application of simulated theory of dynamic systems. The reliability of used model was verificated with comparison of measured and simulated values of sprung mass T815 vehicle accelerations. In the article, there are presented courses of total vertical forces of Quarter-Car Simulation for the first, the second and the fourth classification degree of unevenness, which was considered through the world's respected parameter IRI (International Roughness Index). Obtained simulated dynamic effects of the vehicle on the pavement were used for modification of a relevant Slovak design method of asphalt pavements.
\end{abstract}

\section{Keywords:}

Pavement;

Unavenness;

Dynamic responses of vehicle.

\section{Introduction}

With the reference to [1], the road unevenness is one of the most important pavement characteristic that has significant influence to comfort and road safety and serviceable life of pavement and vehicles. Pavement surfaces have a disordered profile of unevenness, at which stochastic unevenness in little measures one can't see with naked eye. At present, the vehicle pavementdynamic interaction is the most commonly analyzed by mathematic simulations and can be solved for the purpose of determining:

- influence of unevenness to road safety and comfort of driving [1], [2],

- negative impact of vehicle vibration to human health [2],

- dynamic strain of pavements or bridges [3], [4],

- strain of constructional parts of vehicles [5], [6].

In the article, there are presented outputs of simulated dynamic response of heavy vehicle to stochastic unevenness, which are further used as an input for pavement tension calculation. Dynamic response was quantified for three different pavement surfaces from longitudinal unevenness point of view in accordance with determined classification scale of pavement management system.

\section{Simulation of dynamic response of vehicle}

Vertical force effect between the wheel and pavement $F_{\mathrm{k}}(t)$ during moving wheel consists of two parts: the static force $F_{\text {stat }}$ which is proportional to static load of wheel and the time variable dynamic force $F_{\mathrm{dyn}}(t)$ :

$$
F_{k}(t)=F_{\text {stat }} \pm F_{d y n}(t) \text {. }
$$

Dynamic vertical wheel force $F_{\text {dyn }}(t)$ is generated by vibration of moving vehicle, which is kinematically activated by unevenness of pavement surface. Special simulations of dynamic response 
of vehicle were performed by algorithms of quarter car model. This model is mathematically described by the two second-order differential equations, where we must find a vector of spatial derivations:

$$
\begin{aligned}
& \ddot{z}_{s} \cdot m_{s}+C_{s} \cdot\left(\dot{z}_{s}-\dot{z}_{u}\right)+k_{s} \cdot\left(z_{s}-z_{u}\right)=0, \\
& \ddot{z}_{s} \cdot m_{s}+m_{u} \cdot \ddot{z}_{u}+k_{t} \cdot z_{u}=k_{t} \cdot y,
\end{aligned}
$$

where: $m_{s}, m_{u}$ - weight of the sprung mass and the unsprung mass

$k_{s}, k_{t}-$ constant of the linear spring and the tire

$C_{s}$ - coefficient of linear damper

$z_{s}, z_{u}$ - displacement of the sprung and he unsprung mass

$\dot{z}_{s}=d z_{s} / d t, \dot{z}_{u}=d z_{u} / d t-$ vertical velocity of the sprung/unsprung mass

kg],

$\ddot{z}_{s}=d^{2} z_{s} / d t^{2}, \ddot{z}_{u}=d^{2} z_{u} / d t^{2}-$ vertical acceleration of the sprung/unsprung mass

By DYNSIM KCS algorithm (based on algorithm for evaluation of longitudinal unevenness - IRI KCS, according to original methods [7] - [9]), it is possible to get dynamic forces on the contact between the wheel and pavement on the basis known longitudinal profile for arbitrary reference models and simulated speeds. The algorithm consists of the following basic steps:

\subsection{Initial value determining for the first step, $i=1$}

On the basis of discrete data sequence $y_{(i)}, i=1,2,3, \ldots, N$ of elevations of longitudinal profile, it is necessary to continuously evaluate $Z^{\top}(x)$ - vector of spatial derivations of input profile.

$Z^{T}(x)=\left(z_{1 i}, z_{2 i}, z_{3 i}, z_{4 i}\right)$

where: $z_{s i}^{\prime}=z_{1 i}=d z_{s} / d x$ - vertical velocity of sprung mass in the point $i$

$\left[\mathrm{m} \cdot \mathrm{m}^{-1}\right]$

$z_{s i}^{\prime \prime}=z_{2 i}=d^{2} z_{s} / d x^{2}-$ vertical acceleration of sprung mass in the point $i$

$\left[\mathrm{m} \cdot \mathrm{m}^{-2}\right]$

$z_{u i}^{\prime}=z_{3 i}=d z_{u} / d x-$ vertical speed of unsprung mass in the point $i$

$\left[\mathrm{m} \cdot \mathrm{m}^{-1}\right]$

$z_{u i}^{\prime \prime}=z_{4 i}=d^{2} z_{u} / d x^{2}-$ vertical acceleration of unsprung mass in the point $i$

$\left[\mathrm{m} \cdot \mathrm{m}^{-2}\right]$

The following initial values are used for simulation of value of the vector $Z^{\top}(x)$ :

$z_{21}=z_{41}=0$,

$Z_{11}=Z_{31}=\left|\frac{y_{(1+k)}-y_{i}}{k \cdot d x}\right|$,

where: $d x$ - is a size of measuring interval, distance between two consecutive spot heights of longitudinal profile in $\mathrm{m}$ (most often 0.25 or $0.5 \mathrm{~m}$ ) [m],

$k=I N T(0.5 / d t)$,

where: $d t$ - time for transition of distance $d x[s]$.

We can physically interpret these initial conditions as a model start-up time on the middleunevenness pavement surface during the first $0.5 \mathrm{sec}$. of simulated ride time.

\subsection{Calculation of profile slope input}

The profile slope input is computed for every measuring point (we must know elevations of longitudinal profile per $0.25 \mathrm{~m}$ ): 
$y_{(i)}^{\prime}=\left|\frac{y_{(i+1)-y_{(i)}}}{d x}\right|$,

where: $y^{\prime}(i)$ - smoothed profile slope input, $i=2,3, \ldots, N$.

\subsection{Computation of the vector of spatial derivations $Z(x)$}

Computed smooth profile is an input for double-mass quarter car model, and its dynamic response is continuously computed. For computation of the vector of spatial derivations $Z^{\top}(x)(i)=\left(z_{1, i} ; z_{2, i} ; z_{3, i} ; z_{4, i}\right)=\left(z_{s, i}^{\prime} ; z^{\prime \prime}{ }_{s, i} ; z_{u, j}^{\prime} ; z^{\prime \prime}{ }_{u, i}\right)$ is realized by equation (9):

$$
z_{j, i}=\sum_{j=1}^{4} s_{j j} \cdot z_{j, i-1}+r_{j} \cdot y_{i}^{\prime},
$$

where: $j=1,2,3,4$ described vector component,

$\mathrm{i}=1,2, \ldots, \mathrm{N}$ is present step,

$\mathrm{S}=\left[\mathrm{s}_{\mathrm{jj}}\right]-$ state transition matrix $4 \times 4$,

$R=\left[r_{j}\right]$ - partial response matrix $1 \times 4$.

It is possible to solve the matrix $S$ by expansion to Taylor's series by equation:

$\underline{S}=e^{(\underline{A} \cdot d t)}=\underline{I}+\underline{A} \cdot d t+\underline{A}^{2} \cdot d t^{2} / 2 !+\underline{A}^{3} \cdot d t^{3} / 3 !+\ldots$

The DYNSIM KCS algorithm is built for computing state transition matrix with 10 components of series, so in general we can get a following equation:

$\underline{S}=\underline{I}+\sum_{i}^{n} A^{i} \frac{d t^{i}}{i !}$.

For a constant length of the step on which $y^{\prime}(i)$ is a constant, the $S$ and $R$ matrices can be computed from the $A$ and $B$ matrices:

$\underline{S}=e \underline{A \cdot d t}$,

$R=\underline{A}^{-1} \cdot(\underline{S}-\underline{I}) \cdot B$

where: $d t(s)=d x(m) \cdot 3600\left(s \cdot h^{-1}\right) \cdot 0 \cdot 001\left(\mathrm{~km} \cdot \mathrm{m}^{-1}\right) / \mathrm{v}(\mathrm{kph})$, $I$ - identity matrix $4 \times 4$.

$\underline{A}=\left[\begin{array}{cccc}0 & 1 & 0 & 0 \\ -K_{2} & -C & K_{2} & C \\ 0 & 0 & 0 & 1 \\ K_{2} / u & C / u & -\left(K_{1}+K_{2}\right) / u & -C / u\end{array}\right]$,

$B=\left[\begin{array}{c}0 \\ 0 \\ 0 \\ K_{1} / u\end{array}\right]$, 


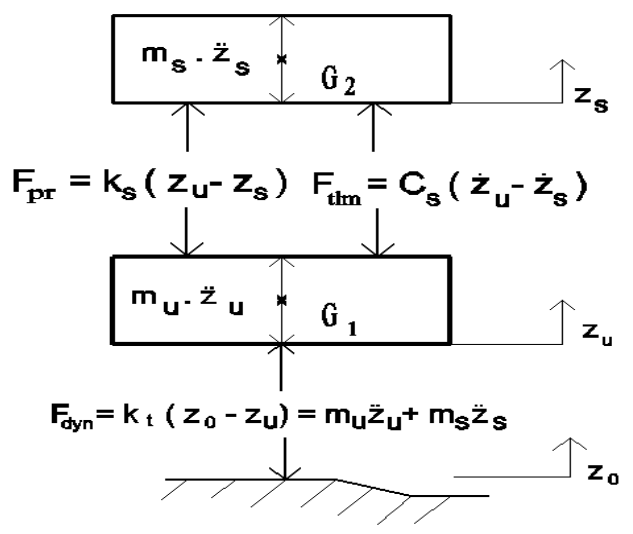

Fig. 1: Schematic representation of dynamic force computing.

\subsection{Determination of a vertical dynamic force}

The principle for finding of vertical force by means of quarter car model is showed in the Fig. 1. There is a lettering in the Fig.1, where index "s“ means sprung masses of a car and index "u" means unsprung masses. Equation (15) represents basic equation for computation of vertical dynamic force, which is an additional load of a pavement.

$$
F_{d y n}=k_{t} \cdot\left(z_{o}-z_{u}\right)=m_{u} \cdot \ddot{z}_{u}+m_{s} \cdot \ddot{z}_{s}
$$

\section{Verification of simulation model}

For verification of model verity was used TATRA 815 C3 S1 vehicle (Fig. 2). Measurement of dynamic response of this vehicle was performed by measurement of equivalent acceleration vibrations level, which were indicated by speed control bump (Fig. 2). On the board of the vehicle, there were placed detectors, the accelerometer NOR-110/13900 with inbuilt third octave filter. There were used two detectors for the acceleration measurement (Fig. 3).

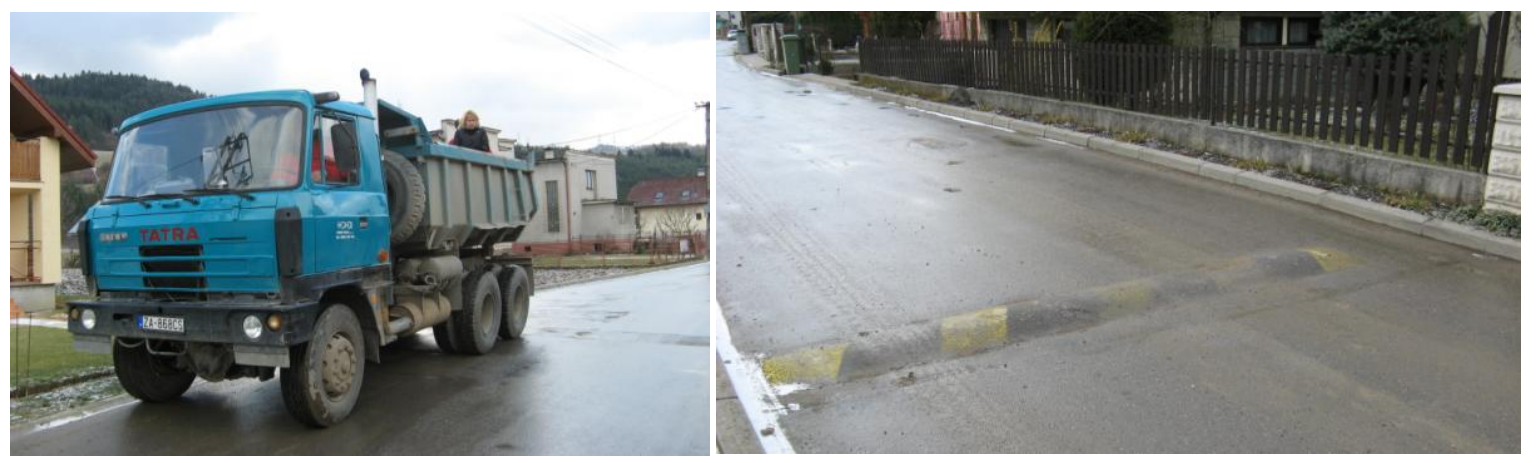

Fig. 2: View of the Tatra T815 C3S1 vehicle and speed control bump on the pavement, height $5 \mathrm{~cm}$, width $50 \mathrm{~cm}$ in Dolný Hričov village.

First of them was located on the axle of the vehicle, which is on the unsprung mass. The other one was located on the board, which is on the sprung mass. Detectors were fastened by a magnet.

Obstruction used for experiment of measurement vibrations was a speed control bump built from asphalt concrete, and its high was $5 \mathrm{~cm}$ and width was $50 \mathrm{~cm}$. Equivalent acceleration vibrations level $L_{a}$ was measured at the time when a vehicle gone through a bump. Measured interval was $10 \mathrm{~ms}$, for accurate capture of accelerations. The vehicle rode at a speed from interval $5-40 \mathrm{~km} \cdot \mathrm{h}^{-1}$ at $5 \mathrm{~km} . \mathrm{h}^{-1}$ a piece. There were performed three rides at each speed for the purpose of making an average. 

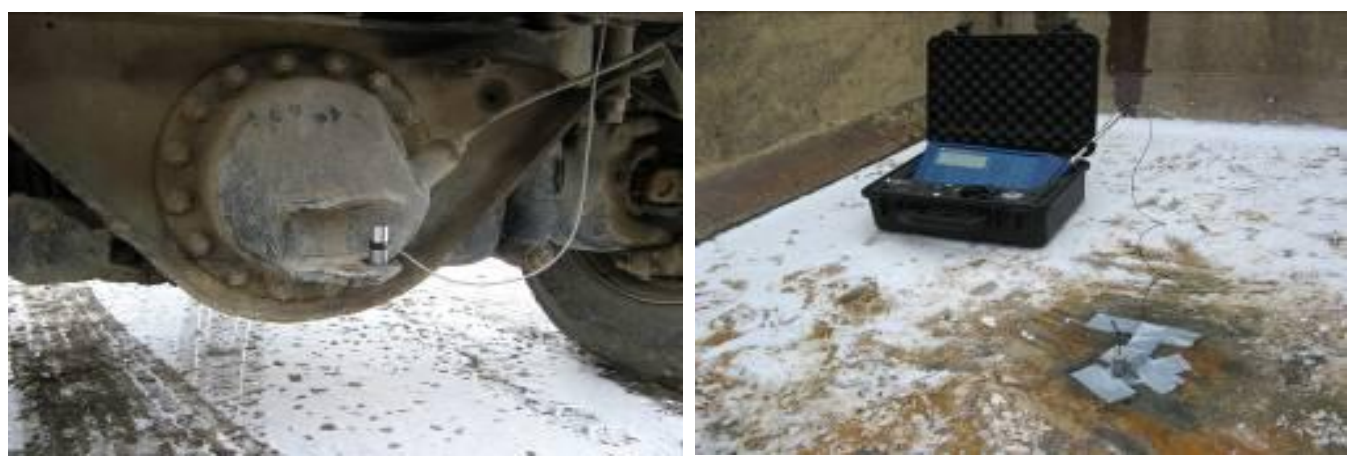

Fig. 3: The location of detectors on the unsprung and sprung mass of vehicle T815.

Equivalent acceleration vibrations level $[\mathrm{dB}]$ was evaluated according to equation:

$L_{a}=20 \cdot \log \frac{a_{e f}}{a_{o}}$

where: $a_{e f}$ - is an acceleration of vibrations

$a_{0}$ - is a reference acceleration of vibrations, $a_{0}=10^{-6} \mathrm{~m} \cdot \mathrm{s}^{-2}$.

An effective value of accelerations of vibrations $\left[\mathrm{m} . \mathrm{s}^{-2}\right] a_{e f}$ is calculated by equation:

$a_{e f}=a_{o} \cdot 10^{\frac{L a}{20}}$.

According to the equation (18), there was calculated the effective value of accelerations of vibrations. After that, there was determined a maximal value of acceleration of vibrations for each ride speed for sprung and unsprung mass as well. Acceleration vibrations level values $L_{a}$ (17) were computed by mathematical function logarithm, and that's why data output from accelerometer are appeared in absolute values. The thin curved line in Fig. 4 shows the effective value of accelerations of vibrations - output data measured by accelerometer placed on vehicle riding over the bump. The thick curve represents these values after adjusting, so we can see a consecutive additional loading and a weight transfer during a ride over the bump.

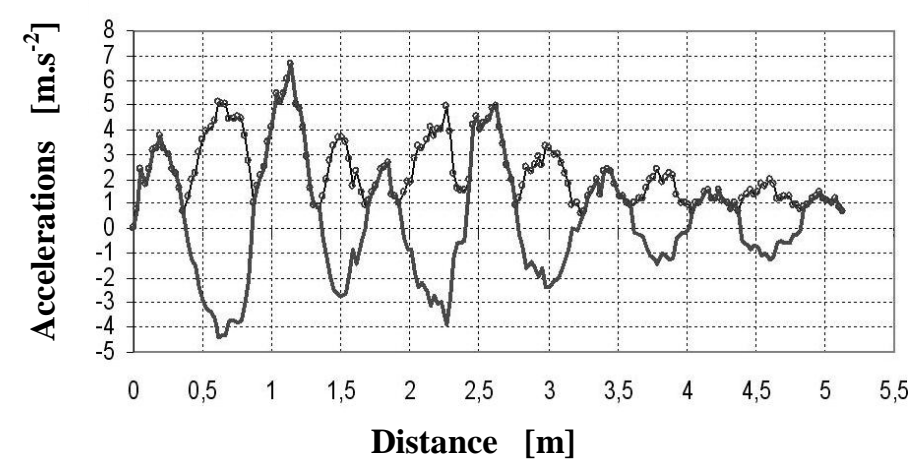

Fig. 4: Values adjusting of accelerations of vibrations by vehicle riding over the bump.

In the DINSIM KCS algorithm, there was performed a simulation with the same conditions and parameters of vehicle as by experimental riding over the speed control bump. There is a huge match in comparison between the simulation curve and the measured one (Fig. 5). 


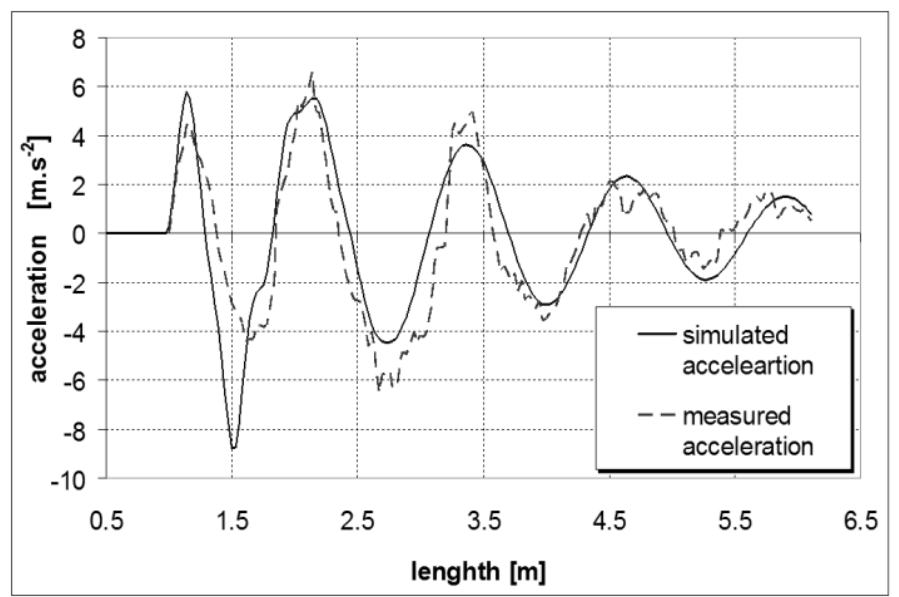

Fig. 5: The comparison between simulated and measured values $T 815 v_{\text {sim }}=10 \mathrm{~km} \cdot \mathrm{h}^{-1}$.

After necessary modification of calculated effective values of acceleration of vibrations that are indicated by real vehicle it can be established, that outputs from the DYNSIM KCS algorithm are comparable with acceleration values determined by experimental measuring. This statement is valid mainly for sprung part of vehicle. After valuation of performed experiment we can say that quarter car model is very appropriate [3]-[7] for simulations made for searching additional loading of road pavements, and results getting from the DYNSIM KCS algorithm are equivalent to dynamic response values indicated by real vehicle ride. The same model is used for pavement condition evaluation within the pavement management system in SR. Generally, there were made simulations for 10 different kinds of pavement surfaces (evaluated by some classification degree of longitudinal evenness [1]-[3]) for each of ride speed of 40,60 and $90 \mathrm{~km} \cdot \mathrm{h}^{-1}$. Obtained output data of dynamic response for second classification degree for ride speed of $60 \mathrm{~km} \cdot \mathrm{h}^{-1}$ are showed in the Fig. 6 .

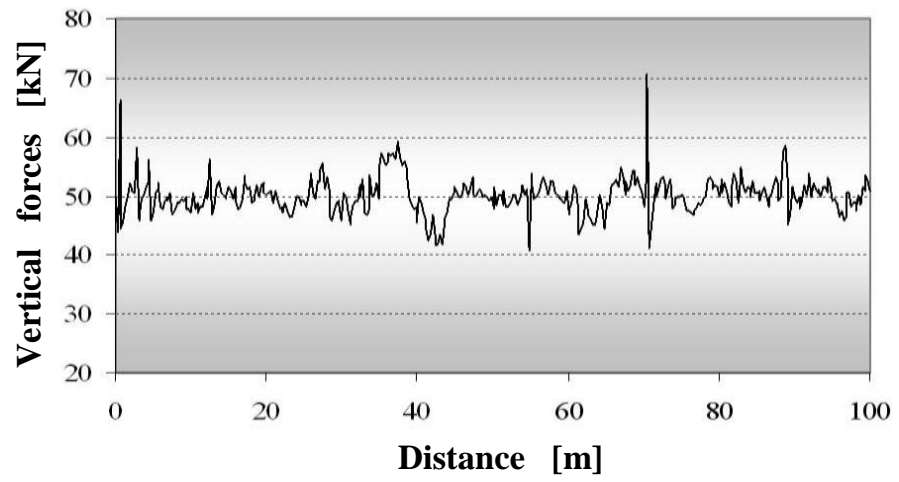

Fig. 6: The course of total vertical force and multiplicity for 2. classification degree of pavement longitudinal evenness.

The course of total vertical force on the pavement with $1^{\text {st }}$ classification degree is between 40 and $60 \mathrm{kN}$, the value of maximum vertical force is $59.24 \mathrm{kN}$ and the value of minimum vertical force is $43.78 \mathrm{kN}$. It means that on this part of the road, road surface unevenness is not clear. The value of maximum vertical force on the pavement with $2^{\text {nd }}$ classification degree is $74.78 \mathrm{kN}$ and the value of minimum vertical force is $24.64 \mathrm{kN}$.

Values of maximum vertical forces obtained by simulation of vehicle transit along road pavement with stochastic unevenness are presented in Fig. 7. There are values in dependence of different classification degrees of pavement longitudinal evenness and in dependence of simulated speeds.

Maximal amplitudes of simulated vertical forces indicated by stochastic pavement unevenness with $2^{\text {nd }}$ classification degree of IRI depending on radial tire stiffness are showed in the Fig. 8. 


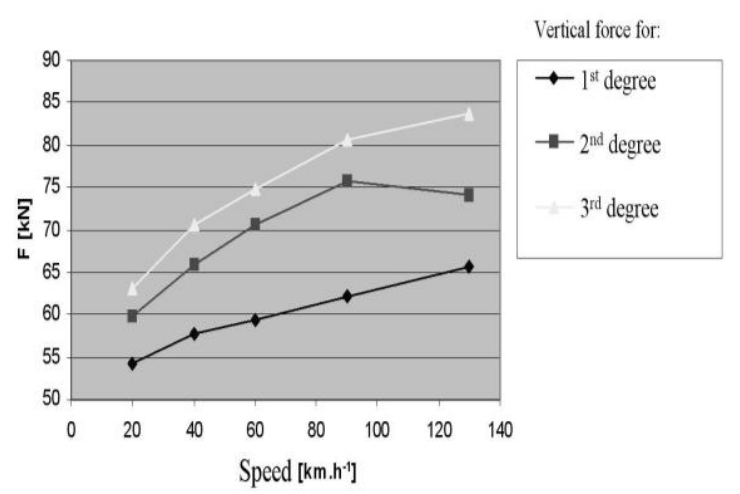

Fig. 7: The dependence of the maximum total vertical force on the simulation speed.

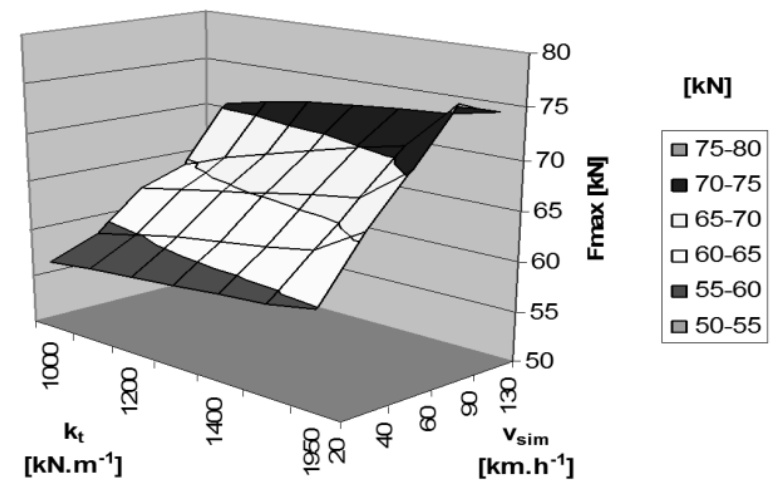

Fig. 8: Maximal amplitudes of vertical dynamic forces for $2^{\text {nd }}$ degree of IRI.

\section{The analysis of stress condition of bitumen pavements from traffic loading}

At the present, it is set a great accent on the valuation of transport effects on the constructions of pavements. Several countries of Europe, because of the protection of pavements before their excess deterioration and abuse, introduce some restrictions for vehicles. Restrictions are for total weights of vehicles, axle load and inflations of tires. The problems with excessive stresses in construction layers of pavements with following precocious degradation of pavements coincide with overloading of vehicles.

\subsection{Equivalent Single Axle Loads}

Equivalent Single Axle Loads (ESAL) is defined with characteristics (Fig. 1) on that are recounting axles of individual type of vehicles. ESAL is usually defined by:

- weight on the axle,

- compression at the contact of tire and pavement,

- number and shape of load areas and their geometric configuration.

Design conditions which are valid in Slovakia define parameters for ESAL according to Fig 9. 


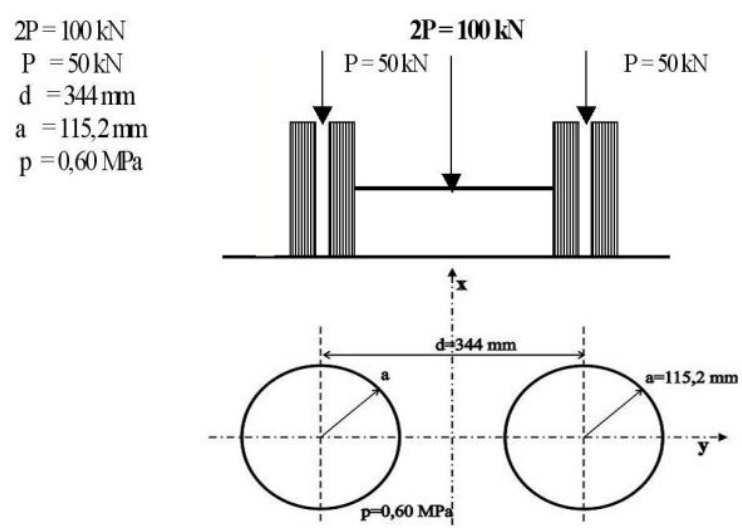

Fig. 9: Computing parameters of ESAL with load of $10 \mathrm{t}$.

\subsection{Appraisal pavement and analysis model}

It is necessary to know that, for the analysis of stresses in the layers of pavements, the size of stress decreases from surface to deep and from axis of weight to sides.

We take into account summer, medium and winter conditions for the proposition of bitumen pavements. According to design method of bitumen pavement there are three climate conditions which are described by relevant temperature:

- summer conditions $27^{\circ} \mathrm{C}$

- medium conditions $11^{\circ} \mathrm{C}$

- winter conditions. $0^{\circ} \mathrm{C}$.

Semi rigid pavement was chosen for observation of changes of stresses in pavement. Structural composition and necessary parameters are in the Table 1. This pavement has consisted of the following layers: asphalt concrete for wearing course (AC $11 \mathrm{O})$, asphalt concrete for base course (AC $16 \mathrm{~L}$ ), asphalt concrete for road base (AC $22 \mathrm{P}$ ), cement stabilization (CBGM $\mathrm{C}_{5 / 6}$ ), crushed aggregate $(\mathrm{CA})$.

Table 1: Parameters of standard semi rigid pavement [12].

\begin{tabular}{|c|c|c|c|}
\hline \multirow{2}{*}{ Layer } & $\begin{array}{c}\text { Thickness } \\
{[\mathbf{c m}]}\end{array}$ & $\mathbf{E l a s t i c ~ m o d u l u s ~ f o r ~ s u m m e r ~ c o n d i t i o n s ~}\left(\mathbf{2 7 ^ { \circ }} \mathbf{C}\right)$ \\
\cline { 3 - 4 } & 5 & 3000 & $\mathbf{v}$ \\
\hline $\mathrm{AC} 11 \mathrm{O}$ & 5 & 2000 & 0.4 \\
\hline $\mathrm{AC} 16 \mathrm{~L}$ & 10 & 1250 & 0.4 \\
\hline $\mathrm{AC} 22 \mathrm{P}$ & 18 & 1200 & 0.44 \\
\hline $\mathrm{CBGM} \mathrm{C}_{5 / 6}$ & 20 & 350 & 0.25 \\
\hline $\mathrm{CA}$ & subgrade & 60 & 0.3 \\
\hline & \multicolumn{3}{|c}{} \\
\hline
\end{tabular}

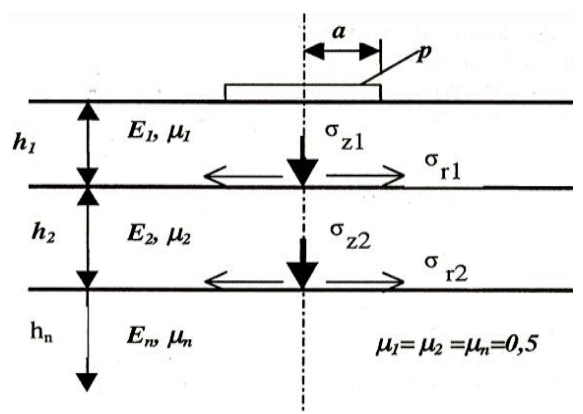

Fig. 10: Multilayered model of pavement structure. 
Calculation of stresses in the layered half-space from effects one and several vertical circle load was realized using the Laymed software [10]. The software works with the model and input data according to Fig. 10. All the presented results were calculated for the input data from Table 1.

\subsection{The stress distribution for two circular loads}

There is examples of radial stresses distributions on the Fig. 11. There are values of radial stresses at the bottom road base (AC $22 \mathrm{P})$.

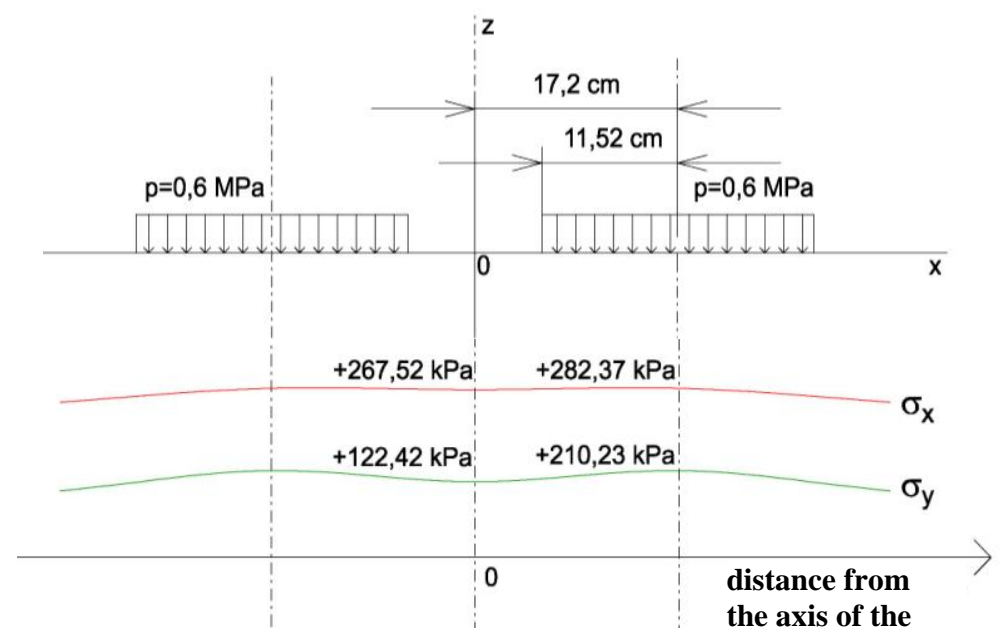

Fig. 11: Radial stresses distribution in the AC $22 \mathrm{P}$ layer.

\subsection{The stress distribution for four circular loads}

There is an example of stress distribution in the road base (AC $22 \mathrm{P}$ ) of the pavement in the Fig. 12 for the pavement loaded by four circular areas. The size of load is $p=0,60 \mathrm{MPa}$.

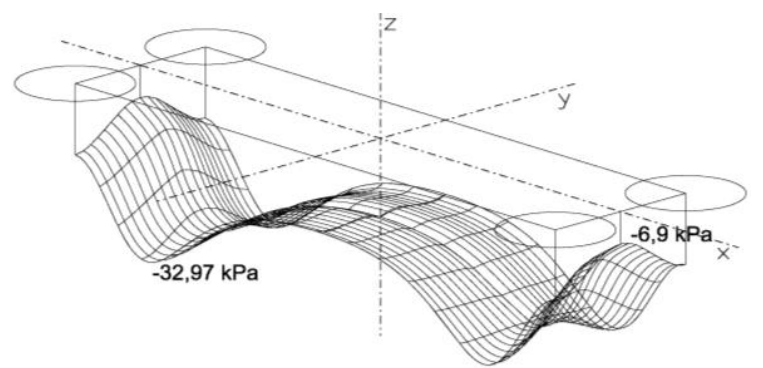

Fig. 12: The stress distribution in the road base $(A C 22 \mathrm{P})$ in the pavement by four of circular Areas [11].

The contact area between the tire and pavement is increased when the load is increasing on the vehicle axle. Moreover, the calculations have shown that radial stresses change depends on the radius of circular load area (Fig. 13). 


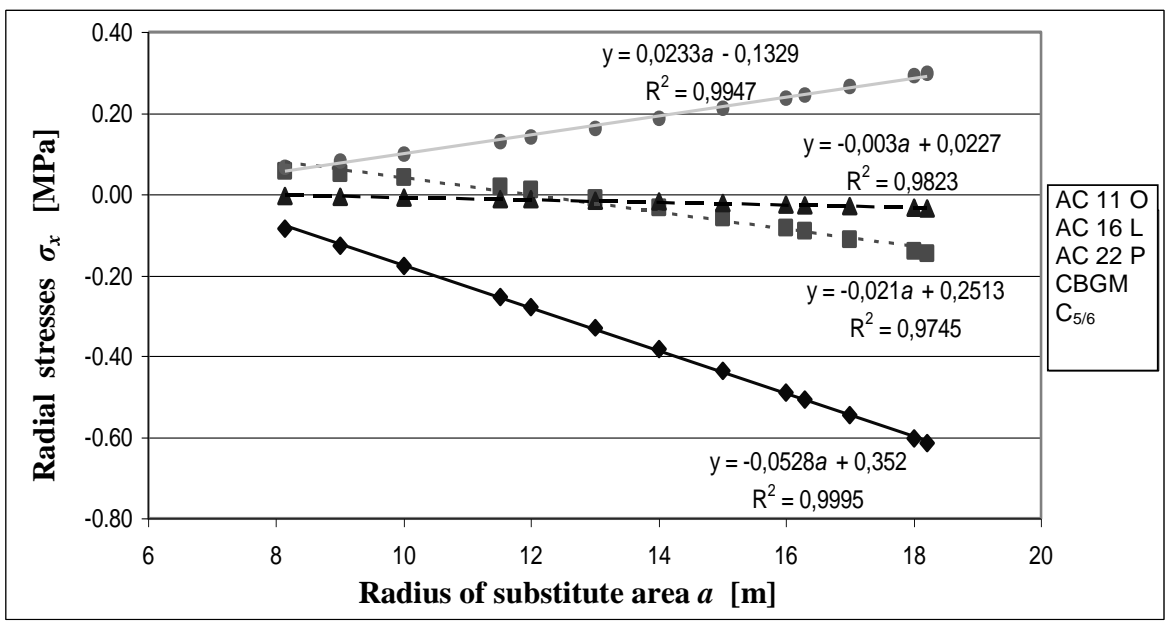

Fig.13:Dependency of radial stresses according to the radius change of substitute circular area at contact compression $\mathrm{p}=0.6 \mathrm{MPa}$.

\section{Conclusions}

Presented results are based on the data sample which contents the real conditions. The verification of the model applicability was verified by comparison of measurements and simulations. For verification of model verity was used TATRA 815 C3 S1 vehicle. Measurement of dynamic response of this vehicle was performed by measurement of equivalent acceleration vibrations level, which were indicated by speed control bump. The stress in pavement depends on the size of loading and on the type of pavement structure, too. When the pavement design is evaluated, the radial stress at the bottom of bitumen layers or cement stabilized layers is critical. The result of calculations has shown that the pass of vehicle with simple rear axle is more unfavorable for pavement than the pass of vehicle with double rear axle, where the load is divided into several contact areas between vehicle and pavement.

\section{Acknowledgement}

The research is supported by European regional development fund and Slovak state budget by the project "Research centre of University of Žilina", ITMS 26220220183.

\section{References}

[1] ČELKO, M. - DECKÝ, M. - KOVÁČ, M.: An analyze of vehicle - road surface interaction for classification of IRI in frame of Slovak PMS. Maintenance and Reliability Nr1(41)/2009. ISSN 1507-2711. pp.15-21.

[2] DECKÝ, M. - KOVÁČ, M.: Longitudinal evenness of road pavements. Scientific monograph (In Slovak), EDIS - University of Žilina 2014, s. 222, ISBN 97880-554-0925-2.

[3] MELCER, J.: Dynamic calculation of highway bridges (In Slovak). EDIS - University of Žilina 1997, p. 287, ISBN 80-7100-4251.

[4] PAPÁNOVÁ, Z. - PAPÁN, D. - KORTIŠ, J.: Microtremor vibrations in the soil experimental investigation and FEM simulation. In: Communications/Komunikácie. Volume 16, Issue 4, 2014, p. 41-47, ISSN 13354205.

[5] MÚČKA, P.: Correlation among Road Unevenness Indicators and Vehicle Vibration Response. In: Journal of Transportation Engineering. Volume 139, issue 8, p. 771-786 DOI: 10.1061/(ASCE)TE.1943-5436.0000558, Published: AUG 12013.

[6] LEITNER, B.: Fatigue Damage Analysis and Fatigue Life Prediction of Lorry Frame under Random Excitation. In: Proceedings of the 18th International Conference TRANSPORT MEANS 2014. P. 9-14, Kaunas Univ Technol, Kaunas, LITHUANIA. OCT 23-24, 2014 Accession Number: WOS:000345692000002, ISSN: 1822-296X. 
[7] SAYERS, M.W. - GILLESPIE, T.D. - QUEIROZ, C.: The International Road Roughness Experiment (IRRE): Establishing correlation and calibration standard for measurements. World Bank, Techn. Paper No. 45, Washington, D.C., 1986.

[8] SAYERS, M., W. - KARAMIHAS, S., M.: The Little Book of Profiling. Basic Information about Measuring and Interpreting Road Profiles. The Regent of the University of Michigan 1998, p. 100.

[9] KARAMISH, S. M. - GILIESPIE, T. D. - KOHN, S. D. - PERERA, R., W.: Guidelines for longitudinal pavement Profile Measurements. University of Michigan, Transportation Research Institute. February 1999, NCHRP Project 10-47, p. 188.

10] NOVOTNÝ, B., HANUŠKA, A.: Theory layered half-space (In Slovak). Veda Bratislava 1983, p. 258.

[11]BLAŽEK, P.: Simulation of dynamic effects of vehicles on the pavement (In Slovak). Dissertation thesis, University of Žilina, Faculty of Civil Engineering, 2008.

[10]DECKÝ, M. et al.: Mechanics road pavements. (In Slovak), EDIS - University of Žilina 2014, p. 494, ISBN 978-80-554-0934-4. 